\title{
Numerical KDV equation by the Adomian decomposition method
}

\author{
M. Akdi, M. B. Sedra
}

Université Ibn Tofail, Faculté des Sciences, Département de Physique, LHESIR, Kénitra, Morocco

\section{Email address:}

msedra@ictp.it (M. B. Sedra)

To cite this article:

M. Akdi, M. B. Sedra. Numerical KDV Equation by the Adomian Decomposition Method. American Journal of Modern Physics. Vol. 2, No. 3, 2013, pp. 111-115 doi: 10.11648/j.ajmp.20130203.13

Abstract: Using the Adomian decomposition method (ADM), we present in this paper a numerical approximation of the solution of the nonlinear KDV equation. The principal task concerns essentially the computation of the Adomian polynomials for this type of equation and thereafter determining a significant criterion to ensure the conditions for convergence of the method.

Keywords: KDV Equation, Numerical Approach, Adomian Decomposition

\section{ADM Method for The KDV Equation}

Consider the following formulation of the $\mathrm{KdV}$ equation:

$$
\begin{aligned}
& \frac{\partial u}{\partial t}+6 u \frac{\partial u}{\partial x}+\frac{\partial^{3} u}{\partial x^{3}}=0, \\
& u(x, 0)=f(x)
\end{aligned}
$$

which can be rewritten as follows:

$$
\frac{\partial u}{\partial t}=-R u-6 N(u), \quad u(x, 0)=f(x)
$$

where $R=\partial^{3} / \partial x^{3}$ represents the linear operator of the equation; and $N(u)=u \partial u / \partial x$ is the non-linear function.

According to the Adomian decomposition, the solution is expressed as:

$$
u(x, t)=\sum_{n=0}^{\infty} u_{n}(x, t)
$$

and the non-linear part by:

$$
N(u)=\sum_{n=0}^{\infty} A_{n}
$$

with:

$$
A_{n}=\frac{1}{n !} \frac{d^{n}}{d \lambda^{n}}\left[N\left(\sum_{i=0}^{\infty} \lambda^{i} u_{i}\right)\right]_{\lambda=0} \text {, pour } n \in \mathrm{N}
$$

By integrating with respect to time and using the initial conditions we have:

$$
u(x, t)=f(x)-\int_{0}^{t}[L u+6 \cdot N(u)] d s
$$

So:

$$
u(x, t)=f(x)-\int_{0}^{t}\left[L\left(\sum_{n=0}^{\infty} u_{n}(x, s)\right)+6 \cdot \sum_{n=0}^{\infty} A_{n}\right] d s .
$$

For the $\mathrm{KdV}$ equation, the Adomian polynomials can be expressed as follows:

$$
A_{n}=\sum_{i=0}^{n} u_{i} \frac{\partial u_{n-i}}{\partial x}
$$

This allows us to deduce $u_{n}(x, t)$, namely:

$$
\left\{\begin{array}{l}
u_{0}(x, t)=f(x) \\
u_{n+1}(x, t)=-\int_{0}^{t}\left[R u_{n}+A_{n}\right] d s, \quad n \geq 0
\end{array}\right.
$$




$$
u(x, 0)=\frac{1}{2} \operatorname{sech}^{2}\left(\frac{x}{2}\right)
$$

We proceed in the following to compute $A_{n}(x, t)$ and $u_{n}(x, t)$ for $n \in[0,10]$ and to be able to determine the approximate solution $\tilde{u}_{n}(x, t)$ up the 10th order using the following formula:

$$
\begin{aligned}
\widetilde{u}_{n}(x, t) & =\sum_{i=0}^{n} u_{i}(x, t) \\
& =f(x)-\int_{0}^{t}\left[L\left(\sum_{i=0}^{n} u_{i}(x, s)\right)+6 \cdot \sum_{i=0}^{n} A_{i}\right] d s .
\end{aligned}
$$

Thus, the approximate solution is :

$$
\tilde{u}_{10}(x, t)=\sum_{i=0}^{10} u_{i}(x, t) .
$$

\section{Numerical Results}

We will develop in the following, a comparison of the graphical presentations between different iterations of the approximate solution to a given order and the exact solution, in a range of space $[-10,10]$ and two different intervals of time $[0,5]$ and $[0,1.5]$, as shown below:

\begin{tabular}{|l|l|l|}
\hline Function & $\begin{array}{l}\text { Graphic Presentation } \\
x \in[-\mathbf{1 0}, \mathbf{1 0}] ; t \in[0,5]\end{array}$ & $\begin{array}{l}\text { Graphic Presentation } \\
x \in[-10,10] ; t \in[0,1.5]\end{array}$ \\
\hline$u_{0}(x, t)$ & & \\
\hline & & \\
\hline$\tilde{u}_{1}(x, t)$ & & \\
\hline & & \\
\hline & & \\
\hline
\end{tabular}

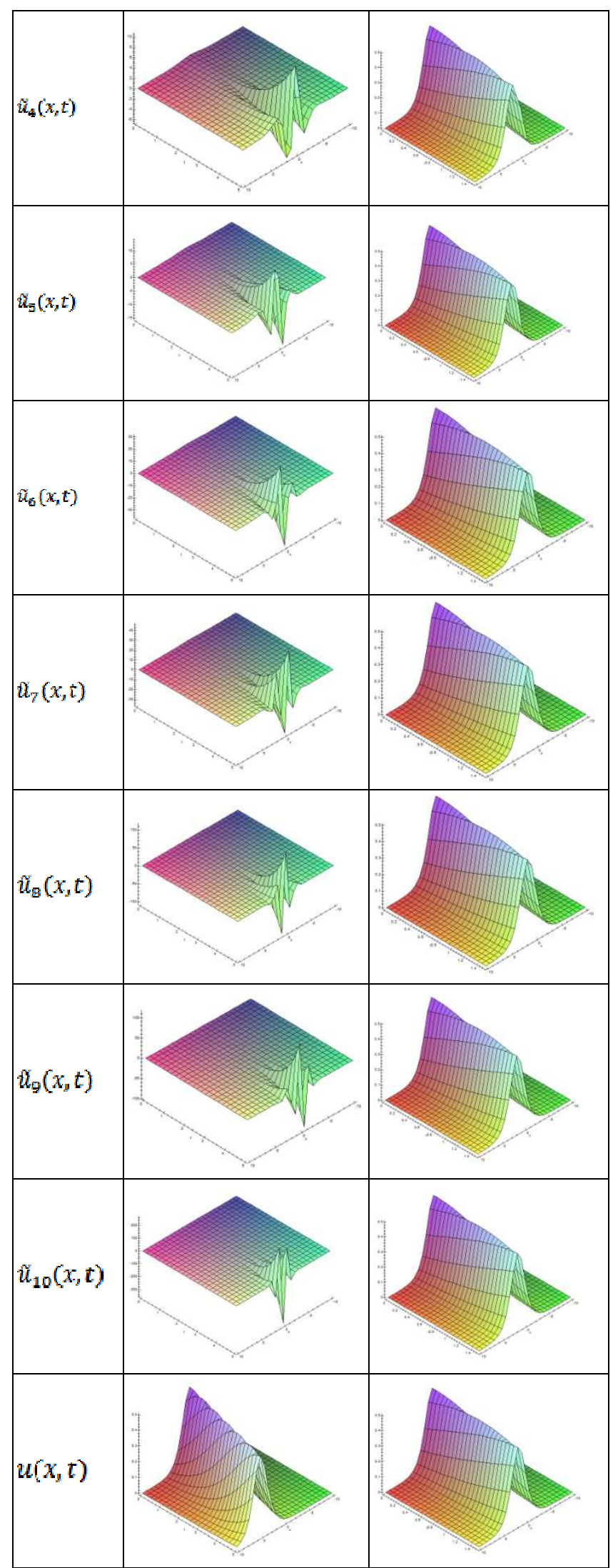

Based on graphical presentations presented above, it should be noted the following observations:

The asymptotic results of the approximate solution, from about $5^{\text {th }}$ order diverging at the growing end of the time 
parameter;

Good agreement between the exact solution and the approximate solution in the reduced interval time $[0,1.5]$.

To emphasize the second remark, we proceed to the graphical presentation of two functions:

$$
\left\{\begin{array}{c}
\mathrm{u}(0, \mathrm{t})=\frac{1}{2} \operatorname{sech}^{2}\left(\frac{\mathrm{t}}{2}\right) \\
\tilde{\mathrm{u}}_{10}(0, \mathrm{t})=\frac{1}{2}-\frac{1}{8} \mathrm{t}^{2}+\frac{1}{48} \mathrm{t}^{4} \\
-\frac{17}{5760} \mathrm{t}^{6}+\frac{31}{80640} \mathrm{t}^{8}-\frac{7369}{154828800} \mathrm{t}^{10}
\end{array}\right.
$$

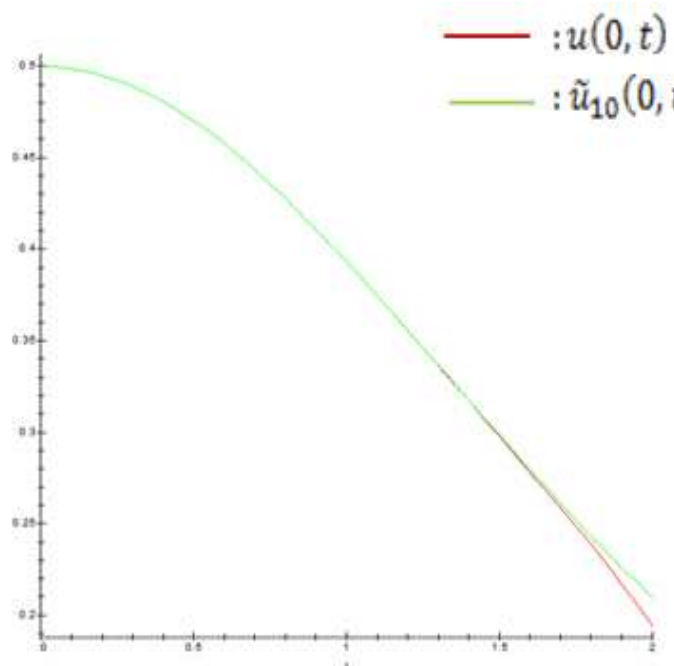

The last presentation clearly shows that in the neighbourhood of the reduced interval time, there are almost coincidence between the two graphs and that beyond it the approximate solution diverges in a sustained manner compared with the exact solution, which can estimate the rate between the maximum values of the two cases to 400 times. We propose to calculate a convenient time value designated by $\tau$, from which the approximate solution $\tilde{u}_{n}(x, t)$ begins to diverge.

\section{Convergence Analysis of the ADM Method for the KDV Equation}

We consider that $N: X \rightarrow X$ is a real analytic function in a $\operatorname{disc} B_{R}(f) \subset X$ whose radius is $R \succ 0$ and $L: X \rightarrow X$ verifies $\|E(t) . f\|_{X} \leq C .\|f\|_{X}$ where $C \succ 0$ and endow $X$ of the norm $\|u\|_{X}=\sup _{u \in X}(|u(x)|)$.

The formulation of Adomian method enables us to write:

$$
u_{n+1}(t)=\int_{0}^{t} E(t-s) \cdot A_{n}\left(u_{0}(s), \ldots, u_{n}(s)\right) \cdot d s, \quad n \geq 0
$$

According to the Cauchy estimation, there exists $R \succ 0$ et $M_{R} \succ 0$ such that:

$$
\left\|\frac{\partial^{k} N(f)}{\partial u^{k}}\right\|_{X} \leq \frac{k ! M_{R}}{R^{k}}, \quad k \geq 0 .
$$

The Taylor series expansion of $N(u)$ for $u=f$ :

$$
N(u)=\sum_{k=0}^{\infty} \frac{1}{k !}\left[\frac{\partial^{k} N(f)}{\partial u^{k}}\right](u-f)^{k},
$$

which converges for all $\|u-f\|_{X} \prec R$ and more, we have:

$$
\begin{aligned}
\|N(u)\|_{X} & \leq \sum_{k=0}^{\infty} \frac{1}{k !} \frac{k ! M_{R}}{R^{k}}\|u-f\|_{X}^{k}, \\
& \leq \frac{M_{R}}{1-\frac{\|u-f\|_{X}^{k}}{R}}=\frac{R \cdot M_{R}}{R-\rho}
\end{aligned}
$$

We define the function:

$$
g(\rho)=\frac{R \cdot M_{R}}{R-\rho}
$$

where:

$$
\rho=\|u-f\|_{X} \prec R
$$

Thus we have:

$$
\left\|\frac{\partial^{k} N(f)}{\partial u^{k}}\right\|_{X} \leq g^{(k)}(0), \quad k \geq 0
$$

Using the formulation of Adomian method we find:

$$
\left\|u_{0}-f\right\|_{X} \leq(C+1) \cdot\|f\|_{X},
$$

$$
\begin{aligned}
\left\|u_{1}\right\|_{X} & \leq \int_{0}^{t}\left\|E(t-s) A_{0}\right\|_{X} d s \leq C \int_{0}^{t}\left\|A_{0}\right\|_{X} d s \\
& \leq C g(0) t=C t \rho^{\prime}(0), \\
\left\|u_{2}\right\|_{X} & \leq \int_{0}^{t}\left\|E(t-s) A_{1}\right\|_{X} d s \leq C \int_{0}^{t}\left\|A_{1}\right\|_{X} d s \\
& \leq C^{2} g^{\prime}(0) g(0) t=C^{2} \frac{t^{2}}{2} \rho^{\prime \prime}(0),
\end{aligned}
$$

$$
\left\|u_{n+1}(t)\right\|_{X} \leq \frac{C^{n+1}}{(n+1) !} t^{n+1} \rho^{(n+1)}(0)
$$

This allows us to deduce that the Adomian series at $X$, are bounded by the convergent series of powers based on 
$\rho(t)=R-\sqrt{R^{2}-2 R M_{R} C t}$, for all $t \in[0, \tau]$, where $\tau \prec \frac{R}{2 M_{R} C}$, in our case study.

So we have :

$$
\left\{\begin{array}{l}
N(u)=u \cdot \frac{\partial u}{\partial x} \\
f(x)=u(x, 0)=\frac{1}{2} \operatorname{sech}^{2}\left(\frac{x}{2}\right),
\end{array}\right.
$$

and using the Cauchy estimate made earlier:

$$
\left\|\frac{\partial^{k} N(f)}{\partial u^{k}}\right\|_{X} \leq \frac{k ! M_{R}}{R^{k}}, \quad k \geq 0 .
$$

We explicit for the case $k=0$ :

$$
\left\{\begin{array}{l}
\left\|\frac{\partial^{k} N(f)}{\partial u^{k}}\right\|_{X}=\|N(f)\|_{X} \\
\frac{k ! M_{R}}{R^{k}}=M_{R}
\end{array},\right.
$$

and we take:

$$
M_{R}=6 .\left\|f \cdot \frac{\partial f}{\partial x}\right\|_{X} .
$$

Let the solution $E(t)$ be the operator associated with the linear Cauchy problem as follows:

$$
\left\{\begin{array}{l}
\frac{\partial v}{\partial t}=L v \\
v(0)=f \in X
\end{array},\right.
$$

such as $v(t)=E(t) . f$ and based on the above, we have:

$$
\|E(t) \cdot f\|_{X} \leq C \cdot\|f\|_{X} .
$$

In the case of the $\mathrm{KdV}$ equation, the linear operator is: $L=\frac{\partial^{3}}{\partial x^{3}}$, and as previously mentioned, using the Fourier transform for the resolution of the linear problem, we find:

$$
v(x, t)=\frac{1}{\sqrt{2 \pi}} \int_{-\infty}^{+\infty} e^{-i \xi^{3} t+i \xi^{k} x} \hat{f}(\xi) d \xi, \quad \forall(x, t) \in R^{2}(31)
$$

where:

$$
\hat{f}(\xi)=\frac{1}{\sqrt{2 \pi}} \int_{-\infty}^{+\infty} e^{-i \xi x} f(x) d x, \quad \forall \xi \in R .
$$

Thus, by identifying and following the expression of the Fourier transform of the solution $v(x, t)$ can be deduced that: $\hat{E}(t)=e^{-i \xi^{3} t}$.

On the other hand, using the Parseval equality, we have:

$$
\begin{aligned}
\|E(t) \cdot f\|_{X}^{2} & =\frac{1}{\sqrt{2 \pi}} \int_{-\infty}^{+\infty}\left(1+\xi^{2}\right) \cdot|\hat{E}(t) \hat{f}|^{2} d \xi \\
& =\frac{1}{\sqrt{2 \pi}} \int_{-\infty}^{+\infty}\left(1+\xi^{2}\right) \cdot|\hat{E}(t)|^{2}|\hat{f}|^{2} d \xi \\
& =\frac{1}{\sqrt{2 \pi}} \int_{-\infty}^{+\infty}\left(1+\xi^{2}\right) \cdot|\hat{f}|^{2} d \xi \\
& =\|f\|_{X}^{2} .
\end{aligned}
$$

Thereby verifying the inequality:

$$
\|E(t) \cdot f\|_{X} \leq C \cdot\|f\|_{X},
$$

with : $C=1$, in this case .

We have also :

$$
\begin{aligned}
\left\|u_{0}-f\right\|_{X} & \leq\left\|u_{0}\right\|_{X}+\|f\|_{X} \\
& \leq\|E(t) \cdot f\|_{X}+\|f\|_{X} \\
& \leq(C+1) .\|f\|_{X} .
\end{aligned}
$$

or by definition, we can write:

$$
R=(C+1) \cdot\|f\|_{X} .
$$

The determination of the threshold convergence in time, equal to $\tau \prec \frac{R}{2 M_{R} C}$ then expressed by:

$$
\tau=\frac{\|f\|_{X}}{6 .\left\|f \cdot \frac{\partial f}{\partial x}\right\|_{X}}
$$

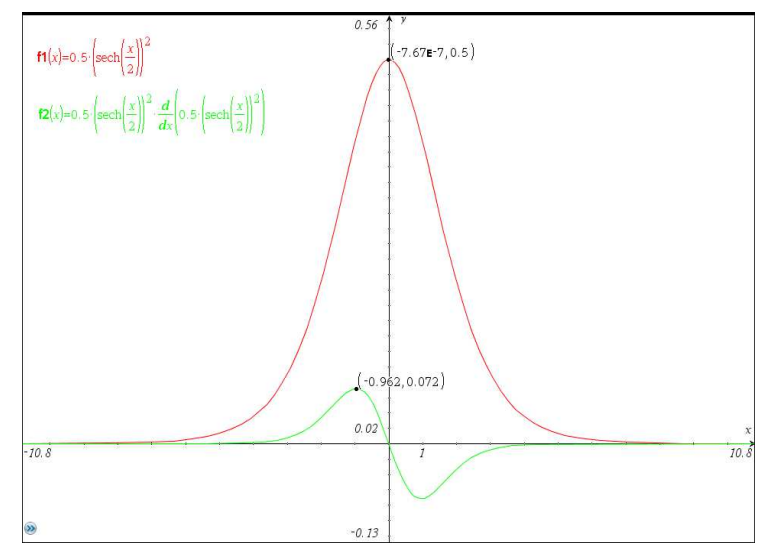

After calculation we find that: 


$$
\tau=1.15
$$

Performing the graphical comparison between the exact solution and the approximated one at the $10 \mathrm{TH}$ order, we find that the detachment between the two curves practically starts from the threshold value calculated and illustrated in the following graphic:

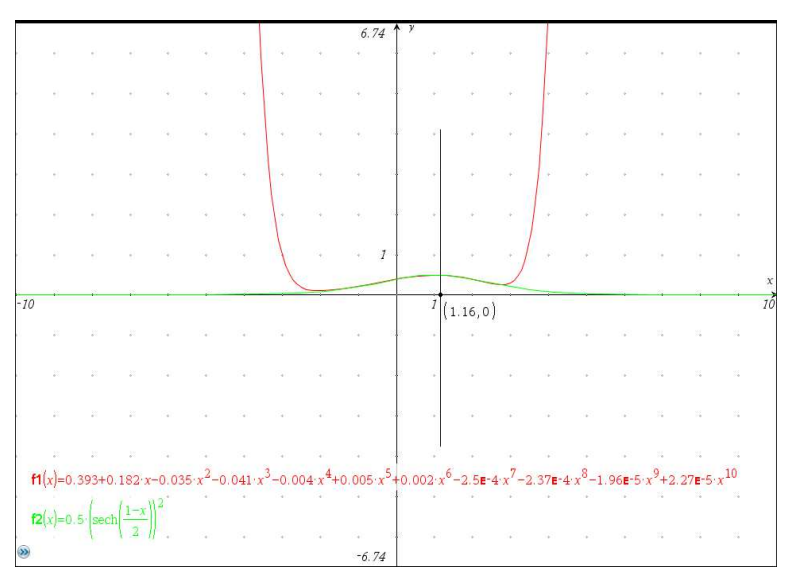

\section{Conclusion}

Through this work, it was possible to apply the ADM method for the resolution of the $\mathrm{KdV}$ equation using a polynomial of time of $10 \mathrm{TH}$ order. Solution that has proven effective in a given time interval. Thus we have completed our study with an analysis, which allowed us to determine the threshold of convergence of the said solution.

\section{References}

[1] K. Abbaoui and Y. Cherruault, Convergence of adomian's method applied to differential equations, Comp Math Appl (1994).

[2] K. Abbaoui and Y. Cherruault, Convergence of Adomian's method applied to nonlinear equations, Math Computer Model (1994).

[3] N. Himoun, K. Abbaoui, and Y. Cherruault, New results of convergence of Adomian's method, Kybernetes (1999).

[4] Y. Zhu, Q. Chang, and S. Wu, A new algorithm for calculating Adomian polynomials, Appl. Math. Comput. (2005).

[5] M. Inc, On numerical solution of partial differential equation by the decomposition method, Kragujevac J Math (2004). 